\title{
Unicast and Multicast IP Error Performance over an ATM Satellite Link
}

\author{
Michael P. Howarth, Haitham Cruickshank, Member, IEEE and Zhili Sun, Member, IEEE
}

\begin{abstract}
IP multicast using ATM-based satellites provides the potential to support multimedia applications, including audio / video streaming and information distribution, at a large scale. This letter considers the impact of satellite channel errors, and equations are derived for the probability of IP datagram loss in the presence of burst errors on the satellite link. When there are a large number of multicast receivers per spotbeam there is a significant probability that one or more recipients will not receive the data, and this has implications particularly for the design of reliable multicast network protocols.
\end{abstract}

Keywords - Internet, Satellite communication, Protocols, Multicast channels, ATM.

\section{INTRODUCTION}

Satellite-based IP services have the potential to deliver multicast services cost effectively. However, their drawbacks include long round-trip delay times and, the particular subject of this letter, their channel error characteristics.

ATM was optimised for operation primarily over fibre optic links. The error performance of this medium is characterised by a low bit error rate, comprising random single bit errors, and few error bursts. In the ATM header the Header Error Control (HEC) field provides protection against single bit errors. Errors that occur in the cell payload are left for higher layers to detect. On a satellite link, errors tend to occur in bursts due to the satellite modem design. These bursts result in a higher cell loss rate than would be expected for the same bit error rate (BER) on fibre optic links.

Loss of an ATM cell results in loss of an IP datagram. For unicast traffic this can cause TCP congestion avoidance algorithms to be invoked, even though the cause was a bit error, not network congestion. For reliable multicast applications the datagram loss can have significant impact if retransmission is required by the multicast protocol.

\section{MODEL}

Our protocol model is shown in Figure 1. A typical satellite communications link employs a convolutional encoding scheme with Viterbi decoding. This improves the effective bit error rate, but means that errors tend to occur in bursts. However, the burst length is limited: for example at an $E_{b} / N_{0}$ of $7 \mathrm{~dB}$, studies have reported no bursts greater than 20 bits in length [1]

Analyses have been performed of ATM performance in a bursty error environment [2,3]. Two key measures are Cell Loss Ratio (CLR), the fraction of cells that are transmitted but not delivered, and Cell Error Ratio (CER), the fraction of delivered cells that have an error in the payload. We assume here that cells are not lost due to buffer overflow, so header errors are the main source of cell loss.

Here we derive expressions for the probability of cell loss and cell error. We assume the length of each error burst follows a Poisson distribution with a mean length $b_{\text {mean }}$ and the burst inter-arrival time is exponentially distributed. We further assume a low BER so that not more than one error burst arrives per ATM cell. Let the burst length be in the range $2 \leq b \leq 40$. The first errored bit of the burst can be in any of the 424 bits of the ATM cell (Figure 1). If the burst starts in any of the bits from 1 to 39 then a cell loss will occur. If the burst starts in bit 40, no cell loss occurs if the HEC algorithm is in correction mode, but the rest of the burst produces a cell error. A burst starting in any of bits 41 to $(424-b+1)$ results in a cell error. A burst starting in bit $(424-b+2)$ causes a cell error and also corrupts one bit of the following cell header; that cell is not lost provided the HEC algorithm is in correction mode. If the burst starts in bits $(424-b+3)$ to 424 then we get a cell error, together with loss of the following cell.

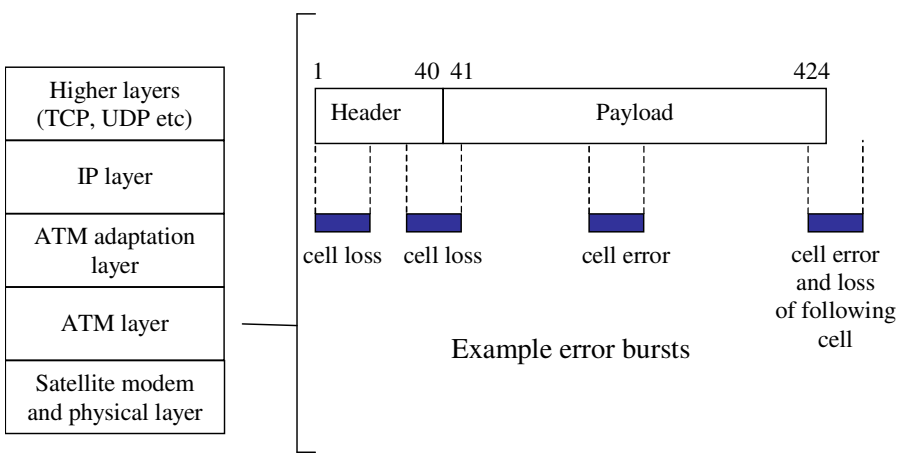

Figure 1: The effect of error bursts on ATM cells

If we assume that the low bit error rate means that the HEC algorithm is in correction mode, the error probabilities are as follows:

$$
\begin{aligned}
& P(\text { no_errors })=P(\overline{\text { loss }} \cap \overline{\text { error }})=1-424 \frac{p}{b} \\
& P(\text { loss } \overline{\text { error }})=39 \frac{p}{b}
\end{aligned}
$$


$P(\overline{\text { loss }}$ ก error $)=(387-b) \frac{p}{b}$

$P($ loss $\cap$ error $)=(b-2) \frac{p}{b}$

where $p$ is the overall bit error rate.

The expressions for the loss and error probabilities should be weighted to reflect the probability distribution of burst lengths $b$, but it is an adequate approximation to assume $b$ is the mean burst length $b_{\text {mean }}$.

Classical IP over ATM is carried using the AAL5 service class (RFC2225). This provides error detection by appending to the SDU padding and an 8-octet trailer with 32-bit CRC checksum. If an error is detected, AAL5 discards the SDU and hence the IP datagram.

An IP datagram will therefore be lost if either a cell loss or a cell error occurs in any ATM cell which is carrying part of the datagram. If the IP datagram is transmitted in N ATM cells, the probability of loss is given by:

$$
P_{\text {IPloss }}=1-P(\text { no errors })^{N}
$$

\section{ANALYTICAL RESUlTS}

Figure 2 shows what may be referred to as the unicast error performance of the satellite link, i.e. the probability of IP datagram loss as a function of BER. The graph is calculated using (5) and assumes a mean burst length of $b_{\text {mean }}=6$ bits [2]. As the mean burst length increases, errored bits are grouped together and affect fewer datagrams, so if $b_{\text {mean }}$ is doubled the probability of datagram loss is approximately halved.

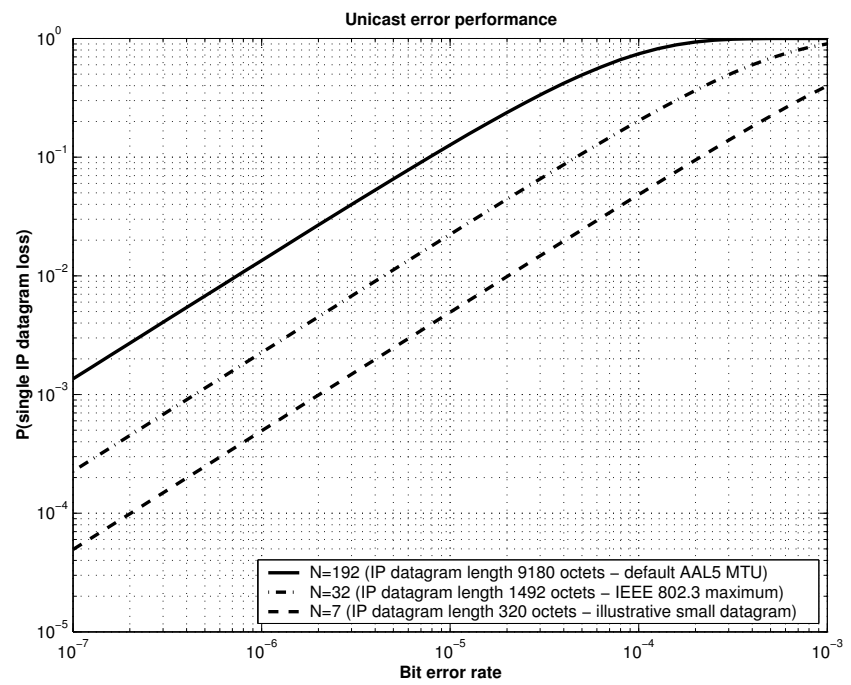

Figure 2: Unicast error performance

Figure 3 shows what may be considered to be the multicast error performance of the satellite link. This is the probability that in a multicast transfer at least one of the recipients does not correctly receive the datagram. For a reliable multicast protocol, this is also effectively the percentage of datagrams that will need to be retransmitted (each retransmission will of course carry with it a further probability of loss which is not considered here). If there are $\mathrm{R}$ multicast receivers per satellite spotbeam then assuming independent losses on the transmission paths the multicast probability of loss is given by:

$$
P_{\text {MulticastIPloss }}=1-\left(1-P_{\text {IPloss }}\right)^{R}
$$

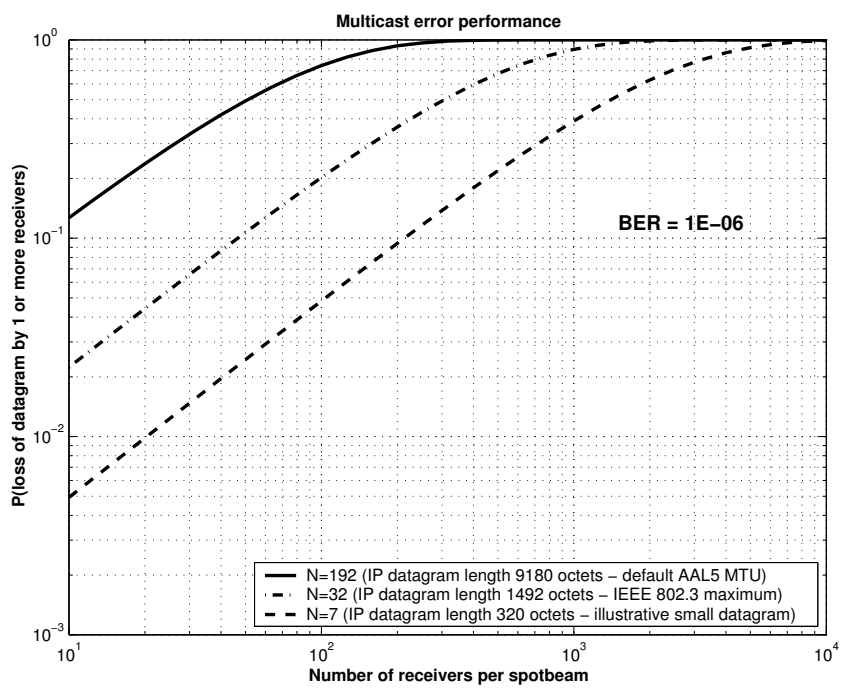

Figure 3: Multicast error performance $\left(10^{-6} \mathrm{BER}\right)$

Figure 3 shows at a bit error rate of $10^{-6}$ that for receiver populations greater than a few tens per spotbeam there is a high probability that any individual multicast datagram will not be received by at least one recipient. For a bit error rate of $10^{-8}$ a receiver population approximately one hundred times larger can be supported for a given error performance.

\section{IMPLICATIONS}

The error performance indicated in Figures 2 and 3 may be acceptable for a streaming multicast transmission since the probability of loss seen by any individual receiver is low, and retransmission is not appropriate. Conversely, a reliable multicast protocol needs to guarantee that data is correctly received at each destination. One option is a separate nonsatellite low data rate link for unicast retransmissions. However, if the retransmission occurs over the satellite link then the delay and throughput reduction will significantly impact the network performance.

When it is expected that applications may employ many receivers per satellite, either the satellite link error performance needs to be improved or the reliable multicast protocol must include appropriate measures. This will reduce the need for ARQ retransmission, and improve link throughput and utilisation.

Options for improving the link error performance include:

- Forward error correction schemes, either at the physical layer (such as the well known concatenated Reed Solomon outer code and convolutional inner code); or data link layer (e.g. transmitting blocks of 
ATM cells with "cell loss detection cells" and parity cells which allow recovery of erased cells [4]);

- Interleaving, either at bit or byte level, spreads an error burst across multiple ATM cells [5,6] so that the HEC correction algorithm can be used to correct errored bits.

A reliable multicast protocol can implement measures such as forward error correction. Some protocols such as RRMP [7] and PGM [8] use burst erasure correction algorithms, but these have not been implemented in other protocols. Alternatively, a scheme like that described above [4] could also be applied to IP datagrams as part of a multicast protocol.

\section{CONCLUSIONS}

We have shown how satellite IP error performance depends on the channel error statistics. When multicast is used to provide services to hundreds of thousands of recipients, transmitted data will be incorrectly received by a significant number of recipients. To rectify this and improve link throughput and utilisation, either the satellite link error performance needs to be improved or the reliable multicast protocol must include error correction mechanisms.

\section{REFERENCES}

[1] J.R. Heissler, Y.A. Barsoum and R. Condello, "An analysis of the Viterbi decoder error statistics for ATM and TCP/IP over satellite communication", Proc. Milcomm 1999, pp.359-363

[2] S. Ramseier and T. Kaltenschnee, "Impact of burst errors on ATM over satellite - analysis and experimental results", COST226 final symposium, Budapest Hungary 10-12 May 1995, pp.99-108.

[3] J.C. Brandão, E.L. Pinto and M.A.G. Maia, "A review of error performance models for satellite ATM networks", IEEE Communications Magazine, July 1999, pp.80-85.

[4] H. Ohta and T. Kitami, "A cell loss recovery method using FEC in ATM networks", IEEE J. Select. Areas Commun., vol. 9, Dec. 1991, pp.1471-1483

[5] D.M. Chitre et al, "Asynchronous transfer mode (ATM) operation via satellite: issues, challenges and resolutions", Int. J. Satellite Communications, vol. 12, 1994, pp.211-222.

[6] W.A. Hamouda and P.J. Mclane, "Performance of ATM cell transmission via regenerative satellite links", IEEE Int. Conf. on Communications 1998, pp.1436-1442.

[7] H.D. Clausen, H. Linder and B. Collini-Nocker, "Internet over direct broadcast satellites", IEEE Communications Magazine, June 1999, pp.146-151.

[8] "PGM reliable transport protocol specification", Internet draft expires 13 August 2001, http://search.ietf.org/internet-drafts/draftspeakman-pgm-spec-06.txt. 